\title{
Frequency and Antibiotic Resistance of Enterobacteriaceae Isolated in Community Urinary Tract Infections at Tebessa Region
}

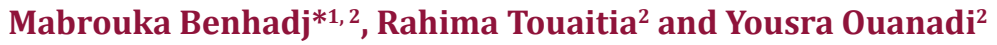 \\ ${ }^{1}$ Department of Biomolecules and Application Laboratory, university of tebessa, Algeria \\ ${ }^{2}$ Departement of Applied Biology, university of tebessa, Algeria
}

Received: 㭗: December 01, 2018; Published: 䒸: December 17, 2018

*Corresponding author: Mabrouka Benhadj, Department of Applied Biology, Faculty of Exact Sciences and Natural and Life Sciences, university of tebessa, Algeria

\begin{abstract}
The objective of the present study was to determine Frequency and antibiotic resistance of Enterobacteriaceae isolated in community urinary tract infections. The study was designed as a prospective study of urine samples from outside hospitals between 1 January and March 2017. Samples with more than $105 \mathrm{CFU} / \mathrm{ml}$ bacteria were considered positive and, for these samples, the bacteria were identified using API 20E and vitek 2 systems. The profile of antibiotic susceptibility was characterized by disc diffusion on Muller Hinton agar. ESBL production was detected by the double disk synergy test. During the study period, 195 Enterobacteriaceae strains were isolated. Among these 195 strains, E. coli was the most common, representing $52 \%$ of the isolates, followed by $K$. pneumoniae (33\%) and other Enterobacteriaceae (12\%). Extended-Spectrum Beta Lactamase (ESBL) producing Enterobacteriaceae significantly increased from seven E. coli strains. Resistance rates to commonly prescribed antibiotics are increasing significantly. All ESBL producing $E$. coli were resistant to cefotaxime, and trimethoprim-sulfamethoxazole that are commonly used for treatment of community-acquired urinary tract infections. ESBL-producing $E$. coli have become major etiological pathogens of community-acquired UTIs in Algeria. Healthcare providers should implement better infection control strategies and careful use of antimicrobials especially in out-patient and community settings.
\end{abstract}

\section{Introduction}

Urinary Tract Infections (UTI) are among the most frequently acquired infections in the community, but also in hospitals and other health care institutions, causing a huge amount of antibiotic consumption [1]. The Enterobacteriaceae, including Escherichia coli, Klebsiella pneumoniae, Proteus mirabilis and other, is the family most commonly implicated in the etiology of urinary tract infections [2]. Most frequently Escherichia coli are isolated in complicated or uncomplicated nosocomial or community acquired urinary system infections [3]. However, the antibiotic susceptibility patterns of Enterobacteriaceae have been constantly changing due to the continuous development of new resistance mechanisms like the production of extended-spectrum beta-lactamases or carbapenemases by bacteria and spread of genes on mobile elements. ESBL-producing bacteria were first reported in the 1983 and are now widespread worldwide. ESBLs confer not only resistance to $\beta$-lactam antibiotics but, oftentimes, cross-resistance to other antibiotics such as aminoglycosides, trimethoprimsulfamethoxazole, and quinolones. Antimicrobial susceptibility studies on uropathogens have been performed in many countries around the world upgrading the medical management of patients with UTIs [4].

\section{Few Studies have Investigated the Prevalence and Risk} Factors Associated with Community-Acquired UTIs

The aim of this study was thus to determine the distribution and susceptibility pattern of bacteria isolated in private laboratory from adult outpatients with urinary tract infections in Tebessa over a 3 months period, and to establish the effects of previous antibiotic use or hospitalization on rates of bacterial resistance, as well as identification of ESBL producer strains among the uropathogens. This study is important for clinician in order to facilitate the empiric 
treatment of patients and management of patients with symptoms of UTIs. Moreover, the data would also help authorities to formulate antibiotic prescription policies.

\section{Material and Methods \\ Clinical Isolates}

A total of 195 non-duplicate strains isolated from patients with urinary tract infections in the period from January 2017 to March 2017 at private laboratory in Tebessa, Algeria, were included in this study. Only strains isolated from outpatients, including patients receiving care at home, were used in the study.

\section{Urinalysis}

The rapid urinary screening tests are urinary sediment, dipstick, and Gram stain. The dipstick test includes $\mathrm{pH}$, proteins, glucose, ketones, blood, bilirubin, urobilinogen, nitrites, leukocyte esterase and urine density. With the urine sediment test, the following data were obtained: bacteria, nitrites, erythrocytes, hemoglobin, epithelial cells and leukocytes.

\section{Isolation and Identification of Organisms}

Samples for urine culture were tested within half an hour of sampling. All samples were inoculated on orientation CHRO Magar and incubated at $37^{\circ} \mathrm{C}$ for $24 \mathrm{~h}$, and for $48 \mathrm{~h}$ in case no growth was observed after $24 \mathrm{~h}$. A specimen was considered positive for UTI in the light of the number of yielded colonies $\left(\geq 10^{5} \mathrm{cfu} / \mathrm{ml}\right)$ and the cytology of the urine through microscopic detection of bacteriuria and PMNs ( $\geq 10$ leukocytes $/ \mathrm{mm}^{3}$ ). Identification of isolates was done up to species level using API 20E kits (bioMerieux SA, France) and vitek 2 system.

\section{Antimicrobial Susceptibility Testing}

Antimicrobial susceptibility testing and interpretation was performed using the vitek 2 system and by the disk diffusion method (Bio-Rad, France) in Mueller-Hinton agar (Bio-Rad) according to the recommendations outlined by the Comité de l'Antibiogramme de la Société Française de Microbiologie CA-SFM 2016. Sensitivity testing was done for Céfotaxim (CTX), Cefoxitin (FOX), Trimethoprim-Sulfamethoxazole (SXT), Ciprofloxacin (CIP), Amikacin (AK), Gentamycin (GN), Nalidixic Acid (NA), Furans (F) Fosfomycin (FF) for each isolate.

\section{Extended Spectrum $\beta$-lactamase Test}

Extended-Spectrum beta-Lactamase production was detected by a Double-Disk Synergy Test (DDST) and was performed by placing disks of ceftazidime, cefotaxime and aztreonam at a distance of $20 \mathrm{~mm}$ (centre to centre) from a disk with amoxicillin/clavulanic acid $(20 / 10 \mu \mathrm{g})$. Enhancement of the inhibition zone between the disks containing clavulanic acid and cefotaxime, ceftazidime or aztreonam indicated the ESBL production [5,6].

\section{Results and Discussion}

During the study period a total of 195 bacterial isolates from community acquired urinary tract infections were consecutively collected. E. coli was the most isolated organism accounting for 100 (52\%). The second commonest isolate was Klebsiella pneumoniae which accounted for 64 (33\%) followed by Pseudomonas aeruginosa (6\%), Proteus mirabilis (5\%), Acinetobacter bumannii (4\%) and Providencia stuartii (1\%) (Figure 1). These findings are in agreement with results found in Constantine hospital (Eastern Algeria) [7] and those reported at the University Hospital Centre of Benimessous in Algiers [8]. In contrast to our results Thirtyeight Enterobacteriaceae isolates including seventeen Klebsiella pneumonia, thirteen Escherichia coli and eight Enterobacter cloacae were recovered from community-acquired urinary-tract infections at four private-laboratories in Bejaia, Algeria, between March 2007 and May 2009 [9]. In comparison with European regions our study showed that E. coli was less frequent than in France 73\% [10] but more frequent than in Ivory Coast [11]. E. coli was the most frequent isolate $(60.64 \%$ of the total isolates) in a study performed in Lebanon [12].

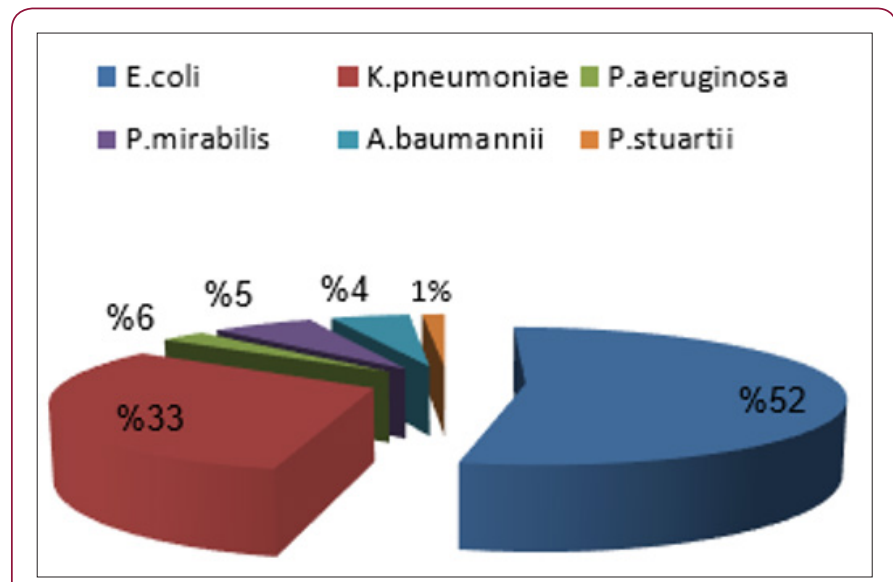

Figure 1: Causative microorganisms for CA-UTI.

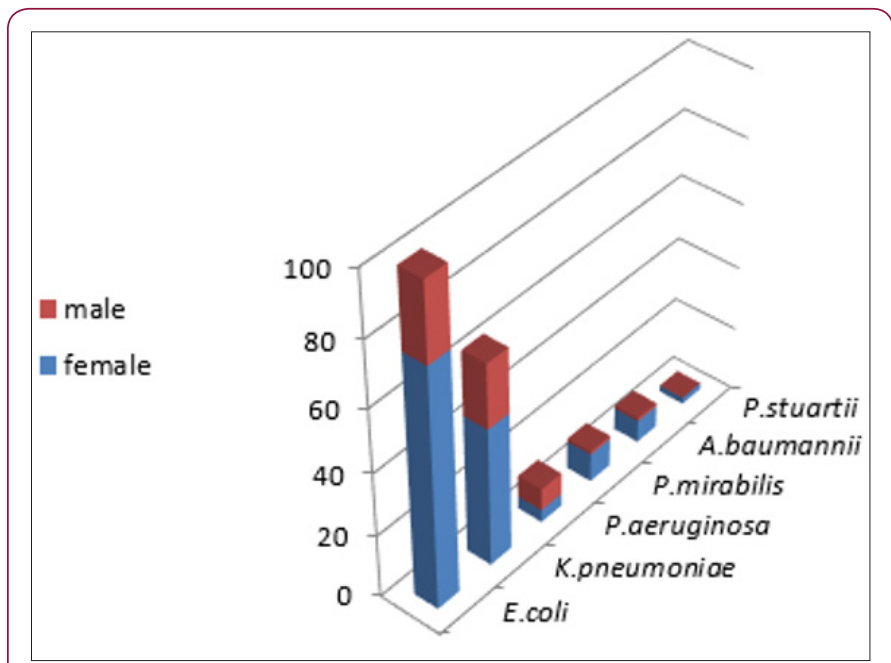

Figure 2: Distribution of species by sex.

Our results about the sex category most affected by Causative microorganisms for CA-UTI. Show that women are most affected urinary tract infection as this is the most common infection (Figure 2). The development of UTIs depends essentially on anatomical factors and on the physiology of urinary tract infection which is usually ascending; there is a strong colonization of the perineum by enterobacteria of digestive origin, in particular E. coli. Our results showed that women are most affected urinary tract infection. 
They are particularly at risk of developing UTIs because of their short urethra, and certain behavioral factors which include delay in micturition, sexual activity and the use of diaphragms and spermicides which promote colonization of the periurethral area with coliform bacteria. Infection in women most often results from perineal or periurethral bacteria that enter the urethra and ascend into the bladder, often in association with sexual activity, or due to mechanical instrumentation such as catheterization [13,14].

The antibiotic resistance rates of the isolates are summarized in Figure 3. Urinary tract infections are a common problem in general practice and are usually treated empirically. Empirical treatments should be based on local data regarding common pathogens and their susceptibility to available antibiotics. The indiscriminate use of antibiotics has developed many resistant microorganisms creating immense clinical problems in the treatment of infections such as those caused by Enterobacteria strains in urinary tract infection [14].

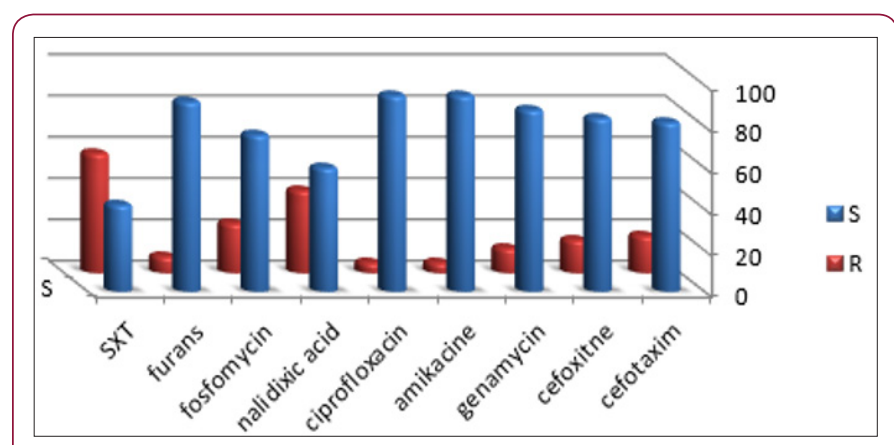

Figure 3: Antibiotic resistance profile of Enterobacteriaceae.

The most of Enterobacteriaceae were susceptible to cefotaxim, cefoxitin (82\%) and amikacine (95\%), while and trimethoprimsulfamethoxazole showed a limited activity (Figure 3). It was noticed that resistance to trimethoprim/sulfamethoxazole and nalidixic acid were significant. Resistance to trimethoprim/ sulfamethoxazole for E. coli $49 \%$, for Klebsiella. $48 \%$ and for P. mirabilis was $42 \%$. Resistance to gentamycin was observed in nine isolates of E. coli, seven isolates of Klebsiella and in five isolates of Acinetobacter bumannii. In this study the highest rate of acquired Resistance of E. coli and other Gram-negative pathogens was observed with trimethoprim/sulfamethoxazole (58\%). High percentage of resistance to this antibiotic was reported in European countries and Brazil, in the range of 39-51\% [15,16].

The resistance rate to fluoroquinolones observed in this study was quite high, particularly in nalidixic acid (40\%), and poses some concerns about their use in empirical treatment of UTIs. Resistance to fluoroquinolones is known to be associated with the previous use of antibiotics, particularly fluoroquinolones, and previous reports have demonstrated that underlying urinary tract diseases predispose patients to repeated UTIs and, in turn, to exposure to antibiotics such as fluoroquinolones [17-19]. Fosfomycin showed activity on $76 \%$ of the strains. On the other hand, $92 \%$ of the strains are classified with sensitivity by testing the furans. These results are relative to the one found in 2015 in France that a low resistance of the strains studied to fosfomycin with (5\%) [10].
In addition, fosfomycin is spared from the multiple resistance mechanisms to which other antibiotics are exposed because of its unique structure and mode of action [20]. This study gives an idea on the rates of antibiotic resistance of the main bacteria involved in urinary tract infections and illustrate the importance of the proper use of antibiotics coupled to surveillance in order to control the spread of these resistances.

\section{Characterization of ESBLs}

The disk diffusion method showed synergy between ceftazidime, cefotaxime, aztreonam, and amoxicillin clavulanic acid in 07 isolates of E. coli suggesting the production of Extended Spectrum Beta Lactamase (ESBL). All ESBL producing E. coli were resistant to cefotaxime, and trimethoprim-sulfamethoxazole. In contrast, these isolates were susceptible to fosfomycin, cefoxitin and furans. All but two of the ESBL producers were resistant to gentamycin. Only two of the seven ESBL producing E. coli isolates showed resistance to amikacine. Regarding quinolones resistance, three isolates were resistant to nalidixic acid and ciprofloxacin (Table 1).

Table 1: Antimicrobial susceptibility profiles in seven ESBL producing E. coli.

\begin{tabular}{|c|c|c|c|c|c|c|c|c|c|}
\hline Strains & CTX & FOX & CIP & GN & AK & NA & FF & F & SXT \\
\hline 01 & R & S & R & R & S & R & S & S & R \\
\hline 02 & R & S & S & S & R & S & S & S & R \\
\hline 03 & R & S & R & R & S & R & S & S & R \\
\hline 04 & R & S & R & S & S & S & S & S & R \\
\hline 05 & R & S & R & R & R & R & S & S & R \\
\hline 06 & R & S & S & R & S & S & S & S & R \\
\hline 07 & R & S & S & R & S & S & S & S & R \\
\hline
\end{tabular}

ESBL producing $E$. coli have been described in hospitals as causing infectious outbreaks, but the presence of this microorganism has also been reported in community [21-24]. During our study, seven ESBL producing E. coli strains were isolated from the urinary tracts of seven non-hospitalized patients in Tebessa country, Algeria.

Most of the ESBL-Enterobacteriaceae isolates were resistant to the other classes of antibiotics, in particular fluoroquinolones and/or cotrimoxazole, which are commonly prescribed by general practitioners, especially for UTIs. Moreover, the high rate of resistance to fluoroquinolones was associated with ESBL production, as indicated by the marked difference in fluoroquinolones resistance observed between ESBL-producing and nonproducing isolates.

\section{Conclusion}

In conclusion the results obtained in this study suggest that $E$. coli is the most common cause of urinary tract infections, followed by Klebsiella pneumoniae. Urinary tract infections occur more often in women than in men. Ciprofloxacin is the most efficient agent against bacterial uropathogens and represents effective option for empirical therapy. ESBL-producing E. coli in urinary isolates is a growing problem and is spreading over the whole country. 
The recognition of the epidemiology and risk factors for ESBLproducing bacteria in the community may affect our management and therapeutic approach. In keeping with our finding that previous antibiotic use was the most important independent risk factor for emergence of ESBL-producing bacteria, further studies and new recommendations that guide management of UTIs and antibiotic use are warranted.

\section{References}

1. Johansen T, Naber KG (2014) Urinary Tract Infections. Antibiotics 3: 375-377.

2. Koksal I, Yilmaz G, Unal S, Zarakolu P, Korten V, et al. (2017) Epidemiology and susceptibility of pathogens from SMART 2011-12 Turkey: Evaluation of hospital-acquired versus community-acquired urinary tract infections and ICU- versus non-ICU-associated intra-abdominal infections. J Antimicrob Chemother 72: 1364-1372.

3. Yılmaz N, Ağuş N, Bayram A, Şamlığlu P, Şirin MC, et al. (2016) Antimicrobial susceptibilities of Escherichia coli isolates as agents of community-acquired urinary tract infection. Turk J Urol 42(1): 32-36.

4. Tansarli GS, Athanasiou S, Falagas ME (2013) Antimicrobial susceptibility of Enterobacteriaceae causing urinary 2 tract infections in Africa: Evaluation of the evidence. Antimicrob Agents Chemother 57(8): 3628-3639.

5. Garrido D, Garrido S, Gutiérrez M, Calvopiña L, Harrison AS, et al. (2017) Clinical characterization and antimicrobial resistance of Escherichia coli in pediatric patients with urinary tract infection at a third level hospital of Quito, Ecuador. Bol Med Hosp Infant Mex 74(4): 265-271.

6. Jarlier V, Nicolas MH, Fournier G, Philippon A (1988) Extended broad spectrum-beta-Iaotamases conferring transferable resistance to newer -lactam agents in Enterobacteriaceae: Hospital prevalence and susceptibility patterns. Infect Dis Rev 10: 867-878.

7. Bouzenoune F, Boudersa F, Bensaad A, Harkat F, Siad N (2009) Urinary tract infections in Ain M'lila (Algeria) Antibiotic resistance of 239 strains isolated between 2006 and 2007. Med Mal Infect 39: 142-143.

8. Aouf A, Gueddi T, Djeghout B, Ammar H (2018) Frequency and susceptibility pattern of uropathogenic Enterobacteriaceae isolated from patients in Algiers, Algeria. J Infect Dev Ctries 12(4): 244-249.

9. Gharout-Sait A, Touati A, Benallaoua S, Guillard T, Brasme l, et al. (2012) CTX-M from community-acquired urinary tract infections in Algeria. J Microbiol Res 6(25): 5306-5313.

10. Malmartel, Ghasarossian C (2016) Epidemiology of urinary tract infections, bacterial species and resistances in primary care in France. Eur J Clin Microbiol Infect Dis 35: 447-451

11. Moroh J-LA, Fleury Y, Tiac H, Bahi C, Lietard C, et al. (2014) Diversity and antibiotic resistance of uropathogenic bacteria from Abidjan. African Journal of Urology 20(1): 18-24.

12. Daoud Z, Salem Sokhn E, Masri K, Matar GM, Doron S (2011) Escherichia coli isolated from urinary tract infections of Lebanese patients between

\section{ISSN: 2574-1241}

DOI: $10.26717 / B J S T R .2018 .12 .002223$

Mabrouka Benhadj. Biomed J Sci \& Tech Res

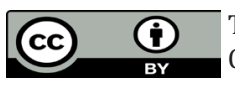

This work is licensed under Creative

Commons Attribution 4.0 License

Submission Link: https://biomedres.us/submit-manuscript.php
2000 and 2009: Epidemiology and profiles of resistance. Front Med (Lausanne) 2: 26.

13. Litza JA, Brill JR (2010) Urinary tract infections. Prim Care 37(3): 491507

14. Ramesh H, Aggarwal KK (2012) Urinary Tract Infections in Women. Indian Journal of Clinical Practice 23(4).

15. Gambogou B, Khadimallah H, Bouacha M, Ameyapoh Y A (2018) Antibacterial activity of various honey monofloral and polyfloral from different regions. J Apither 4(1): 1-8.

16. Nickel CJ (2007) Urinary Tract Infections and Resistant Bacteria: Highlights of a Symposium at the Combined Meeting of the $25^{\text {th }}$ International Congress of Chemotherapy (ICC) and the $17^{\text {th }}$ European Congress of Clinical Microbiology and Infectious Diseases (ECCMID), March 31-April 3, 2007, Munich, Germany. Rev Urol 9(2): 78-80.

17. Cunha MA, Assuncao GLM, Medeiros IM, Freitas MR (2016) Antibiotic resistance patterns of urinary tract infections in a northeastern Brazilian capital. Rev Inst Med Trop Sao Paulo 58: 2.

18. Miliani K, L'Heriteau F, Lacave L, Carbonne A, Astagneau P, et al. (2011) Imipenem and ciprofloxacin consumption as factors associated with high incidence rates of resistant Pseudomonas aeruginosa in hospitals in northern France. J Hosp Infect 77: 343-347.

19. Yasufuku T, Shigemura K, Shirakawa T, Matsumoto M, Nakano Y, et al. (2011) Correlation of overexpression of efflux pump genes with antibiotic resistance in Escherichia coli strains clinically isolated from urinary tract infection patients. J Clin Microbiol 49(1): 189-194.

20. Smithson A, Chico C, Ramos J, Netto C, Sanchez M, et al. (2012) Prevalence and risk factors for quinolone resistance among Escherichia coli strains isolated from males with community febrile urinary tract infection. Eur J Clin Microbiol Infect Dis 31(4): 423-430.

21. Falagas E, Kastoris C, Kapaskelis M, Karageorgopoulos E (2010) Fosfomycin for the treatment of multidrug-resistant, including extendedspectrum B-Lactamase producing, Enterobacteriaceae Infections: A Systematic review. Lancet Infect Dis 10(1): 43-50.

22. Brigante G, Luzzaro F, Perilli M, Lombardi G, Colì A, et al. (2005) Evolution of CTX-M-type beta-lactamases in isolates of Escherichia coli infecting hospital and community patients. Int J Antimicrob Agents 25(2): 157162.

23. Arpin C, Dubois V, Coulange L, André C, Fischer I, et al. (2003) Extended spectrum batalactamase producing Enterobacteriaceae in community and private health care centers. Antimicrob Agents Chemother 47(11): 3506-3514.

24. Rodríguez-Baño J, Navarro MD, Romero L, MartínezMartínez L, Muniain MA, et al. (2004) Epidemiology and clinical of infectious caused by extended-spectrum bata-lactamase producing Escherichia coli in nonhospitalized patients. J Clin Microbiol 42(3): 1089-1094.

25. Rodríguez-Baño J, Navarro MD, Romero L, Martínez Martínez L, Muniain MA, et al. (2004) Epidemiology and clinical of infectious caused by extended-spectrum bata-lactamase producing Escherichia coli in nonhospitalized patients. J Clin Microbiol 42: 1089-1094.

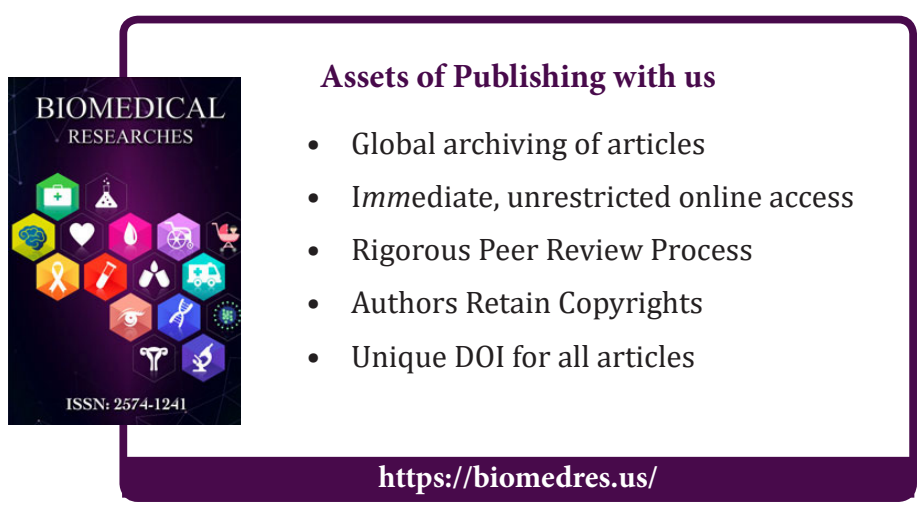

Cite this article: Mabrouka B, Rahima T, Yousra O. Frequency and Antibiotic Resistance of Enterobacteriaceae Isolated in Community Urinary

Tract Infections at Tebessa Region. Biomed J Sci \& Tech Res 12(2)-2018. BJSTR. MS.ID.002223. DOI: 10.26717/ BJSTR.2018.12.002223. 